\title{
Le congrès de I'I.U.F.R.O. à Kyoto (Septembre 1981)
}

\author{
J. PARDÉ
}

L'Union internationale des instituts de recherches forestières (I.U.F.R.O.) fut créée en 1891-1892 à Badenweiler, puis Eberswalde (Allemagne) par un comité de chercheurs allemands, autrichiens, suisses et français spécialement mandatés.

Organisation toujours plus solide et active que jamais, elle s'énorgueillit fort justement en 1982 de ses 394 organisations-membres, regroupant plus de 8000 chercheurs appartenant à 93 nations.

Ses activités scientifiques internationales sont réparties en six «Divisions», articulées en 56 sections (groupes dits «sectoriels» ou «opérationnels»), eux-mêmes subdivisés en 171 groupes de travail $1 *$ !

Les langues officielles de II.U.F.R.O. sont l'anglais, lallemand et le français. Outre de nombreuses réunions partielles qui se succèdent à cadences variables, lUnion intcrnationale tient congrès mondial et général. environ tous les cinq ans.

Prenant la suite du congrès d'Oslo (Norvège) de 1976, le tout récent congrès mondial de 1981 - le $17^{\prime \prime}$ du genre depuis 1893 — se tint au Japon, à Kyoto : les séances en salles (7-12 septembre) étant suivies de nombreuses excursions scientifiques et techniques dans tout le pays (13-17 septembre).

Organisé de façon magistrale en étroite et mutuelle collaboration par le professeur W. LiEse (Allemagne fédérale, président en charge de l'I.U.F.R.O.) et le docteur M. Matsui, directeur de l'Institut national de recherches sur la forêt et les produits forestiers de Tsukuba (Japon), bénéficiant pleinement au Japon de tous les appuis souhaités, ce congrès fut, mieux encore qu'un succès, un véritable triomphe : il sera, à l'avenir, bien difficile non pas de faire mieux, mais d'égaler la réussite de nos collègues japonais.

Le congrès de Kyoto a réuni 1300 participants actifs, venant véritablement du monde entier, auxquels s’étaient jointes 240 «personnes accompagnantes».

Son thème général était : «Les recherches d'aujourd'hui préparent les forêts de demain ».

(*) Par exemple, la division 1 regroupe les activités de recherche relatives à l'Environnement forestier et la Sylviculture.

Parmi ses 9 groupes sectoriels, le groupe S1.02 concerne «le milieu ». S1.02 comprend 9 groupes de travail, dont l'un, S1.02.06, a pour titre - et pour thème : "Classement des stations». 
Tenu dans le magnifique palais des congrès internationaux de Kyoto, il fut ouvert solennellement par son Altesse Impériale le prince hériticr et la princesse son épouse.

En 155 sessions et séances de travail, 330 rapports officiels (parce que «demandés 》 à leurs auteurs) ont été présentés et discutés avec animation et parfois passion. Sy sont ajoutées 330 contributions «volontaires». De plus, quelque 250 «posters » (c'est-àdire des tableaux exposés, expliquant en quelques courtes phrases, graphiques simples, et belles photos tel ou tel thème de recherche) ont été aussi affichés et commentés : cette forme nouvelle simplifiée de communication internationale a été très appréciée.

Les actes publiés du congrès ont nécessité 8 volumes totalisant 3874 pages imprimées !

La qualité scientifique et mondiale du congrès de Kyoto est certaine. Labondance des réunions de petite dimension - 20 à 40 chercheurs - rassemblant les spécialistes, et eux seuls, d'un sujet de recherche bien centré, y fut pour beaucoup. A ces réunions sajoutaient les multiples conversations circonstancielles entre chercheurs, de tous horizons et de tous pays, qui n’étaient pas les moins enrichissantes.

Il nous paraît opportun de citer ici les «résolutions» du congrès, dont il fut ensuite donné largement connaissance à tous les pays membres de II.U.F.R.O.

Ces résolutions s'articulent en «considérations» d'abord, suivies d'une « déclaration $\gg$ solennelle :

\section{Considérations}

Le bois est la principale matière première renouvelable de ce monde, et ses usages s'accroissent en même temps qu'augmentent la population et les niveaux de vie.

La surface terrestre consacrée à la production de biens et services est limitée, et la partie de cette surface octroyée à la forêt est en diminution constante ;

Si l'on ne prend pas de mesures appropriées, l'exploitation sans cesse accrue des forêts mettra en difficulté dans des nombreuses régions non seulement l'approvisionnement futur des biens et services d'origine forestière, mais aussi les ressources en gênes forestiers, la production agricole, les ressources en eau, et l'environnement humain ;

Les surfaces forestières régionales ne sont plus en rapport avec les populations humaines concernées, et il existe certaines incompatibilités entre les écosystèmes forestiers d'origine naturelle et ceux créés par l'homme : ceci est spécialement vrai en certaines régions tropicales.

Le potentiel d'augmentations de la productivité actuelle des forêts est considérable, de même que sont importantes les possibilités d'intégration de demandes très variées de biens et services qui peuvent être formulées.

La recherche forestière est nécessaire au développement du potentiel forestier mondial. Elle est aussi nécessaire pour surmonter les contraintes et difficultés existantes pour soulager la sous-nutrition, la pauvreté et le chômage dont souffrent les hommes dans tant de parties du monde. 


\section{Déclaration}

Le premier devoir de la recherche forestière est daboutir à une compréhension complète des écosystèmes forestiers mondiaux, daccroitre leur productivité tant en quantité qu'en qualité et d'améliorer les taux de conversion et utilisation des produits forestiers. L'affaire revêt un caractère d'urgence toute particulière en régions tropicales.

On devrait encourager les recherches interdisciplinaires ayant pour but une intégration des utilisations diversifiées des terres, spécialement dans le cas des régions tropicales.

La recherche forestière devrait tenir compte des facteurs sociaux, culturaux, opérationnels et économiques, tels qu'ils se présentent dans les différentes régions.

Il existe un besoin important de recherche pour produire du bois de feu dans les régions déboisées, et aussi pour mieux utiliser la biomasse à produire de l'énergie partout dans le monde.

Il faudrait promouvoir une étroite coopération entre hommes politiques, gestionnaires de forêts et chercheurs forestiers, tant au plan national qua plan international, pour garantir une formulation réaliste et fructueuse des programmes de recherches et une mise en pratique effective des résultats de la recherche.

Malgré les difficultés à dégager les crédits nécessaires, les gouvernements et les organisations de soutien de programmes devraient mettre en ouvre des moyens financiers suffisants affectés aux recherches forestières, afin de permettre à ces recherches d'apporter une contribution véritablement effective aux progrès de l'humanité.

$$
*
$$

Enfin, le «conseil international» de l'I.U.F.R.O. renouvela pour moitié à Kyoto - comme de règle à chaque congrès - Ie bureau exécutif de I'Union. Lce nouveau président est le professeur Dusan Mi.INsek, professeur de sylviculture à l'Université de Ljubljana (Yougoslavie). en 1986 .

C'est dans cette ville que se tiendra le 19" congrès mondial de I'I.U.F.R.O.,

D ici là, de nombreuses réunions «partielles» sont prévues un peu partout dans lc monde, notamment en France. Nous les recommandons à lattention de tous ceux qu intéressent les progrès des sciences forestières, et aussi les contacts internationaux.

\section{J. PARDÉ}

ancien coordonnateur de la Division 6 de l'I.U.F.R.O., représentant de la France au Conseil international de l'I.U.F.R.O,. I.N.R.A./C.N.R.F., Champenoux, 54280 Seichamps. 